\title{
Sea Ice Trends in Climate Models Only Accurate in Runs with Biased Global Warming $\mathscr{O}$
}

\author{
ERICA ROSENBLUM AND IAN EISENMAN \\ Scripps Institution of Oceanography, University of California, San Diego, La Jolla, California
}

(Manuscript received 15 June 2016, in final form 21 April 2017)

\begin{abstract}
Observations indicate that the Arctic sea ice cover is rapidly retreating while the Antarctic sea ice cover is steadily expanding. State-of-the-art climate models, by contrast, typically simulate a moderate decrease in both the Arctic and Antarctic sea ice covers. However, in each hemisphere there is a small subset of model simulations that have sea ice trends similar to the observations. Based on this, a number of recent studies have suggested that the models are consistent with the observations in each hemisphere when simulated internal climate variability is taken into account. Here sea ice changes during 1979-2013 are examined in simulations from the most recent Coupled Model Intercomparison Project (CMIP5) as well as the Community Earth System Model Large Ensemble (CESM-LE), drawing on previous work that found a close relationship in climate models between global-mean surface temperature and sea ice extent. All of the simulations with 1979-2013 Arctic sea ice retreat as fast as observations are found to have considerably more global warming than observations during this time period. Using two separate methods to estimate the sea ice retreat that would occur under the observed level of global warming in each simulation in both ensembles, it is found that simulated Arctic sea ice retreat as fast as observations would occur less than $1 \%$ of the time. This implies that the models are not consistent with the observations. In the Antarctic, simulated sea ice expansion as fast as observations is found to typically correspond with too little global warming, although these results are more equivocal. As a result, the simulations do not capture the observed asymmetry between Arctic and Antarctic sea ice trends. This suggests that the models may be getting the right sea ice trends for the wrong reasons in both polar regions.
\end{abstract}

\section{Introduction}

In comprehensive climate model simulations of longterm climate change, individual models are often used to carry out multiple simulations that differ only in their initial conditions. The spread among the simulations approximates the range of possible realizations of internal variability in the climate system. Therefore an individual simulation would not typically match the observations on decadal time scales even if the model were perfect, but the observations are expected to fall within the range of the ensemble of simulations.

Modeling groups from around the world have contributed to each phase of the Coupled Model Intercomparison Project (CMIP). In the third phase (CMIP3; Meehl et al. 2007), virtually none of the models simulated a summer

Supplemental information related to this paper is available at the Journals Online website: http://dx.doi.org/10.1175/ JCLI-D-16-0455.s1.

Corresponding author: Erica Rosenblum, ejrosenb@ucsd.edu
Arctic sea ice cover that diminished as fast as in the observations under historical natural and anthropogenic climate forcing (Stroeve et al. 2007). However, Stroeve et al. (2007) suggested the possibility that the observed Arctic sea ice retreat may represent a rare realization of internal variability that would be captured in only a small fraction of simulations. The CMIP3 models simulated sea ice trends that were more consistent with observations in the Antarctic than in the Arctic (Stroeve et al. 2007; IPCC 2007).

In the current phase (CMIP5; Taylor et al. 2012), the simulated rate of Arctic sea ice retreat is closer to the observations (Stroeve et al. 2012; IPCC 2013). The cause of this reduction in model bias is analyzed in a companion paper (Rosenblum and Eisenman 2016). The ensemble-mean Arctic sea ice trend in CMIP5 is still slower than observed (Stroeve et al. 2012; IPCC 2013), but the observations fall within the range of simulations

Publisher's Note: This article was revised on 20 July 2017 to include the correct supplemental file. An older version was mistakenly included when originally published. 

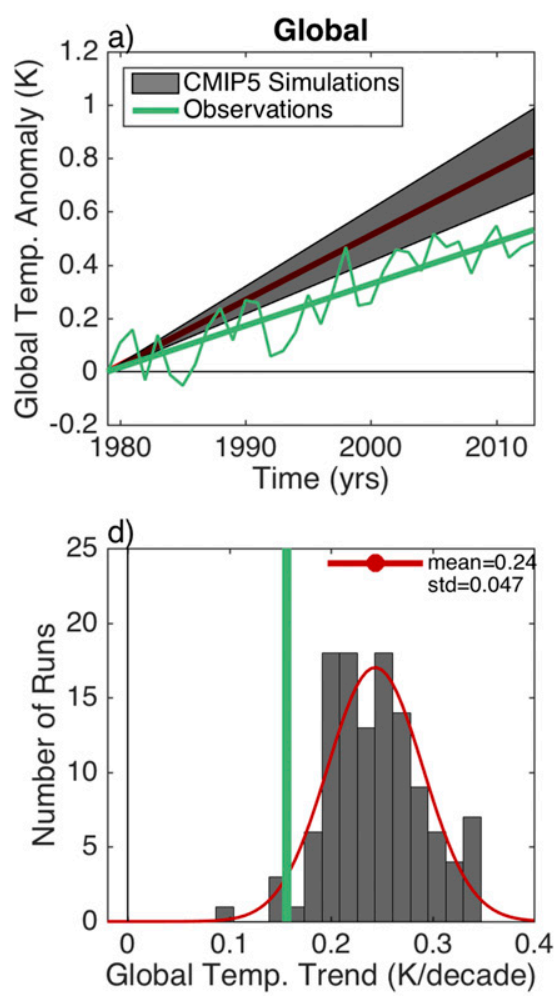
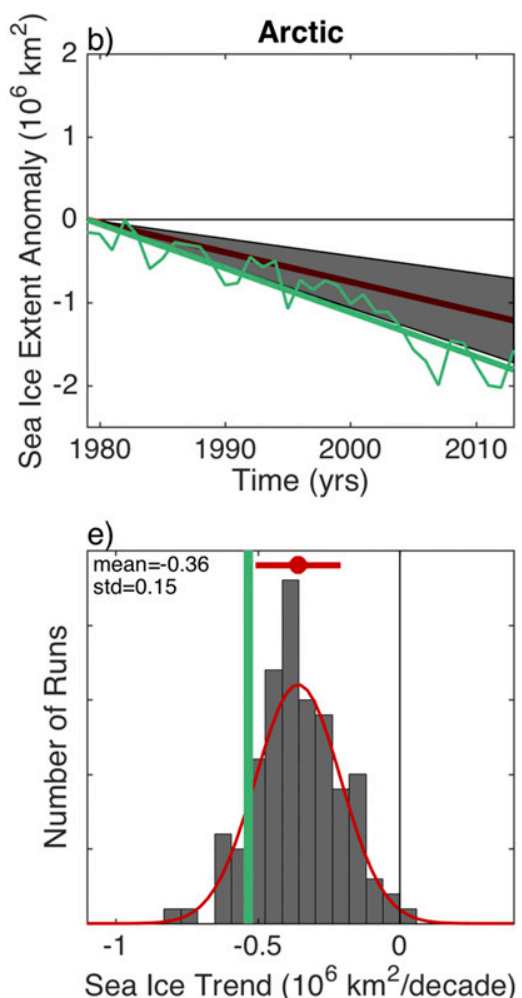
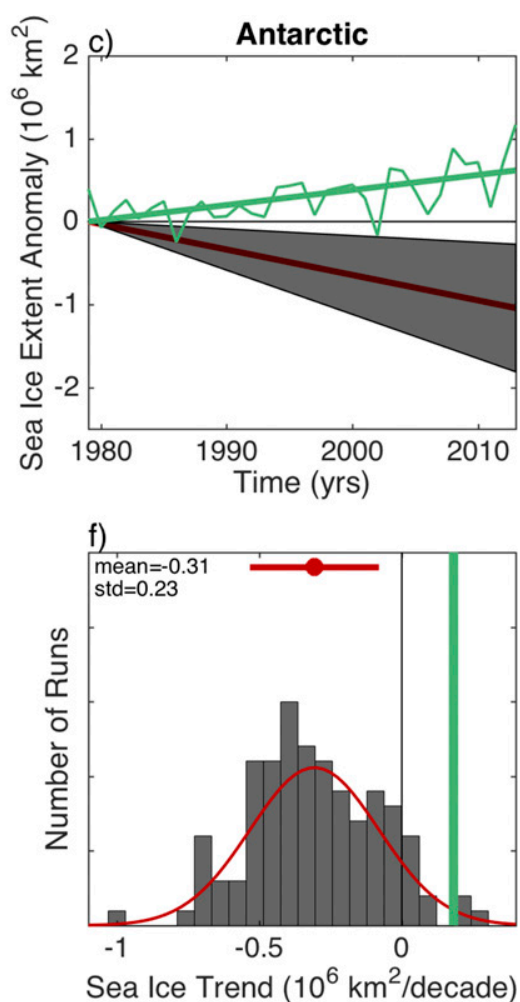

FIG. 1. Observed and CMIP5 modeled linear trends in annual-mean (a),(d) global-mean surface temperature, (b),(e) Arctic sea ice extent, and (c),(f) Antarctic sea ice extent. In (a)-(c), the trends are illustrated as straight lines shifted vertically so that the trend lines go through zero in 1979. The dark red lines indicate the ensemble-mean trend and the gray shadings indicate one standard deviation among the 118 CMIP5 trends. The observed time series and associated trend are also included for each quantity (green). (bottom) Histograms showing the distributions of CMIP5 modeled trends with the observed trend indicated by a green line in each panel. The standard deviation of each distribution around the ensemble mean is indicated by a red error bar above the histogram, and a Gaussian fit to each distribution is plotted in red.

(Figs. 1b,e). Consistent with this, a number of recent studies have suggested that the Arctic sea ice retreat simulated in the current generation of climate models is consistent with observations when simulated internal climate variability is taken into account (Kay et al. 2011; Stroeve et al. 2012; IPCC 2013; Notz 2014; Swart et al. 2015). Given the CMIP5 ensemble-mean results, this would imply that climate forcing has caused some of the observed Arctic sea ice retreat, with the remainder caused by decadal-scale internal variability.

During the past several years, the observed trend toward Antarctic sea ice expansion has become substantially larger and crossed the threshold of statistical significance (Comiso and Nishio 2008; IPCC 2013), which was related to a recent update in the way the satellite sea ice observations are processed (Eisenman et al. 2014). Most CMIP5 models do not simulate this trend (Figs. 1c,f) (e.g., Turner et al. 2013; Zunz et al. 2013; IPCC 2013), contributing to a consensus view that there is "low confidence" in the scientific understanding of the observed Antarctic sea ice expansion (IPCC 2013).
Nonetheless, a number of recent studies have argued that the models are still at least marginally consistent with observations when the range of internal climate variability is considered (Turner et al. 2013; Swart and Fyfe 2013; Zunz et al. 2013; Mahlstein et al. 2013; Polvani and Smith 2013; Goosse and Zunz 2014; Gagné et al. 2015; Fan et al. 2014; Turner et al. 2015; Purich et al. 2016; Jones et al. 2016). In this view, the observed Antarctic sea ice expansion is the result of internal climate variability overwhelming the sea ice retreat that would have occurred due to climate forcing.

Consequently, these recent studies suggest that simulated internal variability can explain the differences between typical state-of-the-art climate model simulations and observed sea ice trends in both the Arctic and the Antarctic. However, a number of previous studies have found that Arctic sea ice extent is approximately linearly related to global-mean surface temperature in climate models (Gregory et al. 2002; Winton 2011; Mahlstein and Knutti 2012; Stroeve and Notz 2015). This suggests that it may be important to consider 
global-mean surface temperature trends when comparing sea ice trends with observations.

Here, we examine the relationship between globalmean surface temperature and sea ice extent in each hemisphere in all available CMIP5 simulations of years 1979-2013, and we compare this with observations. There are 40 different climate models, many of which submitted multiple simulations with differing initial conditions, leading to a total of 118 ensemble members. Hence the CMIP5 ensemble members differ due to both intermodel differences and realizations of internal variability. To isolate the influence of internal variability alone, we also consider simulations from the Community Earth System Model Large Ensemble (CESM-LE) (Kay et al. 2015), which includes 30 ensemble members that are all generated with the same model and differ only in initial conditions. See Table S1 in the online supplemental material for a list of models, and see appendix A below for details regarding the processing of the simulation output and the observations.

Some previous studies have focused on the September or March sea ice trend, whereas others have considered the annual-mean trend. Here we focus on annual-mean trends, thereby averaging over seasonal variability that may be unrelated to long-term changes.

\section{Observed and simulated sea ice trends}

As a starting point, we consider the extent to which the observations lie within the distribution of CMIP5 simulated 1979-2013 Arctic and Antarctic sea ice trends. Each distribution approximates the range of sea ice trends allowed by internal climate variability and differences in model physics. Examining each hemisphere individually, we find that in both cases the observed sea ice trend lands within the overall range of the CMIP5 distribution (Figs. 1e,f).

To quantify the level of agreement, we determine the number of simulated trends that are at least as far in the tail of the CMIP5 distribution as the observed trend. We find that 13 of the 118 simulations have Arctic sea ice retreat at least as fast as the observations and 3 of the 118 simulations have Antarctic sea ice expansion at least as fast as the observations (Table 1). This implies that if the CMIP5 models are correct, then the probability that the Arctic sea ice would retreat as fast as observations is $11 \%$, and the probability that the Antarctic sea ice would expand as fast as observations is $2.5 \%$. These results are approximately similar to previous studies that found that, after accounting for simulated internal variability, the models and observations are statistically consistent in the Arctic (Stroeve et al. 2012; Notz 2014; Swart et al. 2015) and marginally consistent in the
TABLE 1. Fraction of runs with simulated sea ice trends that are at least as extreme as the observations using the distribution of CMIP5 simulated trends (see section 1), effective trends (see section 4), and a pseudo-ensemble of 35-yr periods that have similar levels of global warming to the observations (see section 5). The first column is the fraction with Arctic sea ice retreat as rapid as the observations, and the second column is the fraction with Antarctic sea ice expansion as rapid as the observations. There are 118 simulations of 1979-2013 in the CMIP5 ensemble analyzed here and 1232 overlapping 35 -yr periods in the pseudo-ensemble. Percentages are included to aid in comparison between the rows.

\begin{tabular}{lcc}
\hline \hline & Arctic & Antarctic \\
\hline $1979-2013$ trends & $13 / 118(11 \%)$ & $3 / 118(2.5 \%)$ \\
$1979-2013$ effective trends & $0 / 118(0 \%)$ & $0 / 118(0 \%)$ \\
Pseudo-ensemble & $1 / 1232(0.08 \%)$ & $45 / 1232(3.7 \%)$ \\
\hline
\end{tabular}

Antarctic (Swart and Fyfe 2013; Turner et al. 2013; Zunz et al. 2013; Purich et al. 2016; Jones et al. 2016).

As an alternative method of assessing the level of agreement, we also consider Gaussian fits of the model distributions. This will be useful later in the analysis when the observations fall deep within the tail of the distributions. We find that $12 \%$ of runs in the Gaussian distribution in Fig. 1e have Arctic sea ice retreat at least as fast as the observations, and $1.6 \%$ of runs in the Gaussian distribution in Fig. 1f have Antarctic sea ice expansion as fast as the observations, similar to the raw percentiles given above. It should be noted, however, that these distributions are not expected to be exactly Gaussian. They would be Gaussian, for example, if the simulated sea ice retreat were a linear trend in time at the same rate in all of the ensemble members with superimposed internal variability taking the form of realizations of white noise (e.g., Santer et al. 2008). Under this construction, the center of the distribution is the response to climate forcing, and the width of the distribution represents the influence of internal variability.

\section{Sea ice scales with global temperature}

Previous studies have found an approximately linear relationship in many climate model simulations between global-mean surface temperature and sea ice extent in the Arctic (Gregory et al. 2002; Winton 2011; Mahlstein and Knutti 2012; Stroeve and Notz 2015) and Antarctic (e.g., Armour et al. 2011). The regression coefficient between these two variables is often referred to as the "sea ice sensitivity" to global warming (Winton 2011). We find that this applies to Arctic sea ice in the CESMLE and CMIP5 ensembles (Fig. S1a in the online supplemental material): The annual-mean Arctic sea ice extent and annual-mean global-mean surface air temperature have an ensemble-mean correlation of -0.99 in 

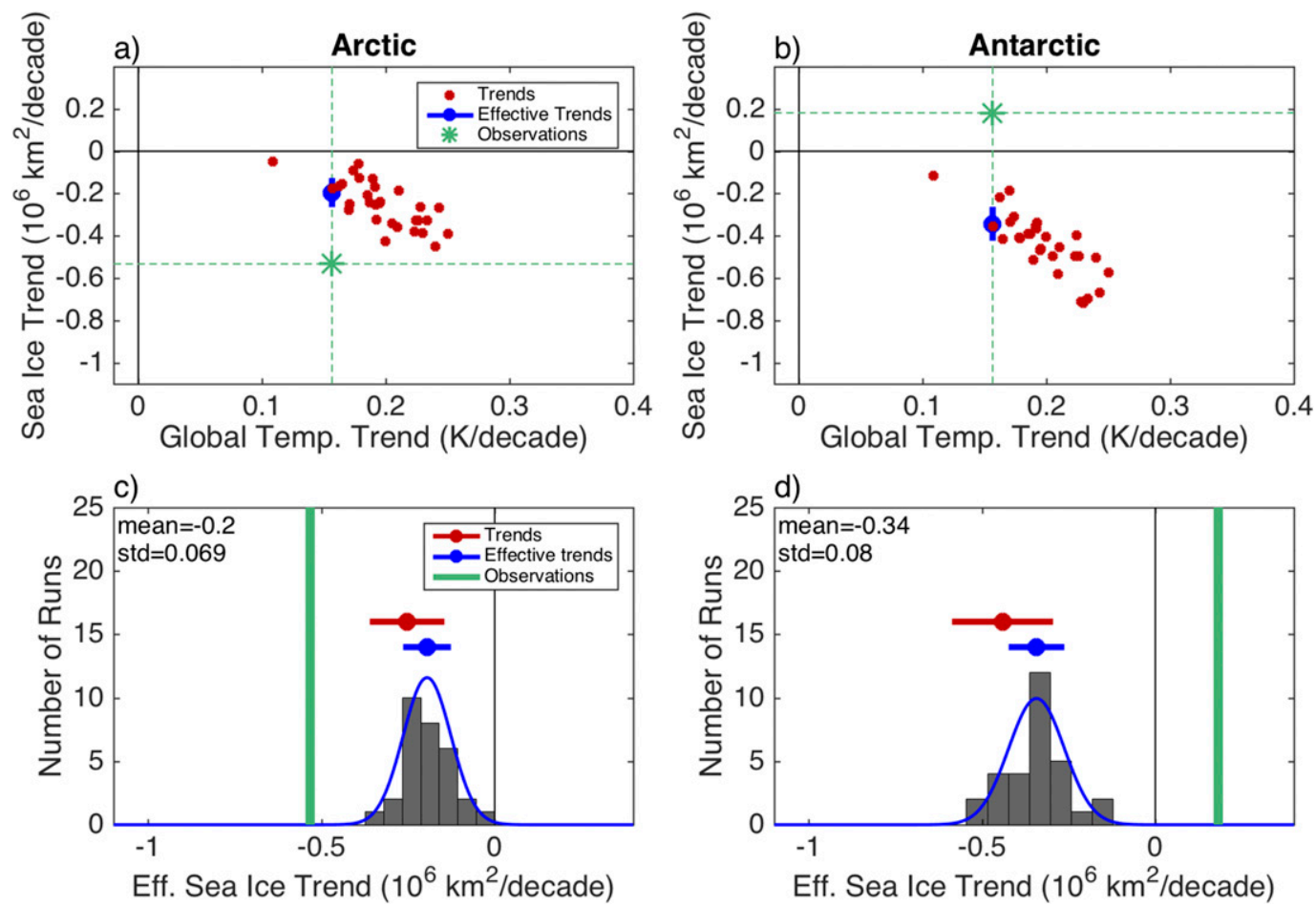

FIG. 2. CESM-LE annual-mean sea ice trends in (a) the Arctic and (b) the Antarctic plotted vs the global-mean surface temperature trend for each ensemble member (red points) with the observations indicated by green dashed horizontal and vertical lines. (c),(d) The distribution of simulated effective sea ice trends (see text for details) from each CESM-LE simulation, with the observed sea ice trend indicated by a green vertical line. The mean and standard deviation of the distribution of simulated sea ice trends (red error bars, repeated from Figs. 1e,f) and effective sea ice trends (blue error bars) are shown, as well as Gaussian fits to the effective sea ice trend distributions (blue curves). The means and standard deviations of the effective trends are repeated for comparison in the top panel (blue vertical error bars).

the CESM-LE simulations of 1920-2100 (Fig. S1c) and -0.94 in the CMIP5 simulations of 1900-2100 (Fig. S1e) (see appendix A for details). We find that the Antarctic sea ice extent has a similar relationship with global temperature (Fig. S1b), although the correlation is somewhat smaller at -0.98 and -0.86 in CESM-LE and CMIP5, respectively (Figs. S1d,f). These relationships imply that simulated 35-yr global-mean surface temperature trends are related to sea ice trends in both hemispheres (scatter of black points in Figs. S2 and S3a,b).

Although this study focuses primarily on CMIP5, we begin by using CESM-LE in order to assess how this relationship influences the distribution of 1979-2013 sea ice trends in realizations of a single model. In Figs. 2a and $2 \mathrm{~b}$ we plot the Arctic and Antarctic sea ice extent trend in each CESM-LE simulation versus the simulated trend in global-mean surface air temperature. This shows a clear relationship in which realizations of internal climate variability that have anomalously large levels of global warming during 1979-2013 also tend to have anomalously large levels of sea ice retreat during this period in both hemispheres. Two representative runs are plotted in Fig. S4 to further illustrate this point. This is consistent with Xie et al. (2016), who found that simulated internal variability in global-mean surface temperature correlates substantially with temperatures in both polar regions.

The results in Fig. 2 are also relevant to the recent study of Notz and Stroeve (2016), who propose a physical mechanism by which sea ice extent responds linearly to cumulative $\mathrm{CO}_{2}$ emissions. This mechanism implies that the previously noted relationship between sea ice extent and global-mean surface temperature is actually an artifact of global temperature also depending linearly on cumulative $\mathrm{CO}_{2}$ emissions. Since the CESM-LE simulations in Fig. 2 each represent identical cumulative $\mathrm{CO}_{2}$ emissions (i.e., each has identical forcing) but have a range of different global-mean temperature trends, they provide an ideal testing ground for this hypothesis. Hence the relationship between global-mean surface temperature trends and sea ice trends in Fig. 2 represents a counterargument to the hypothesis that sea ice extent is fundamentally driven by cumulative $\mathrm{CO}_{2}$ emissions (Notz and Stroeve 2016). Rather, the results in Fig. 2 

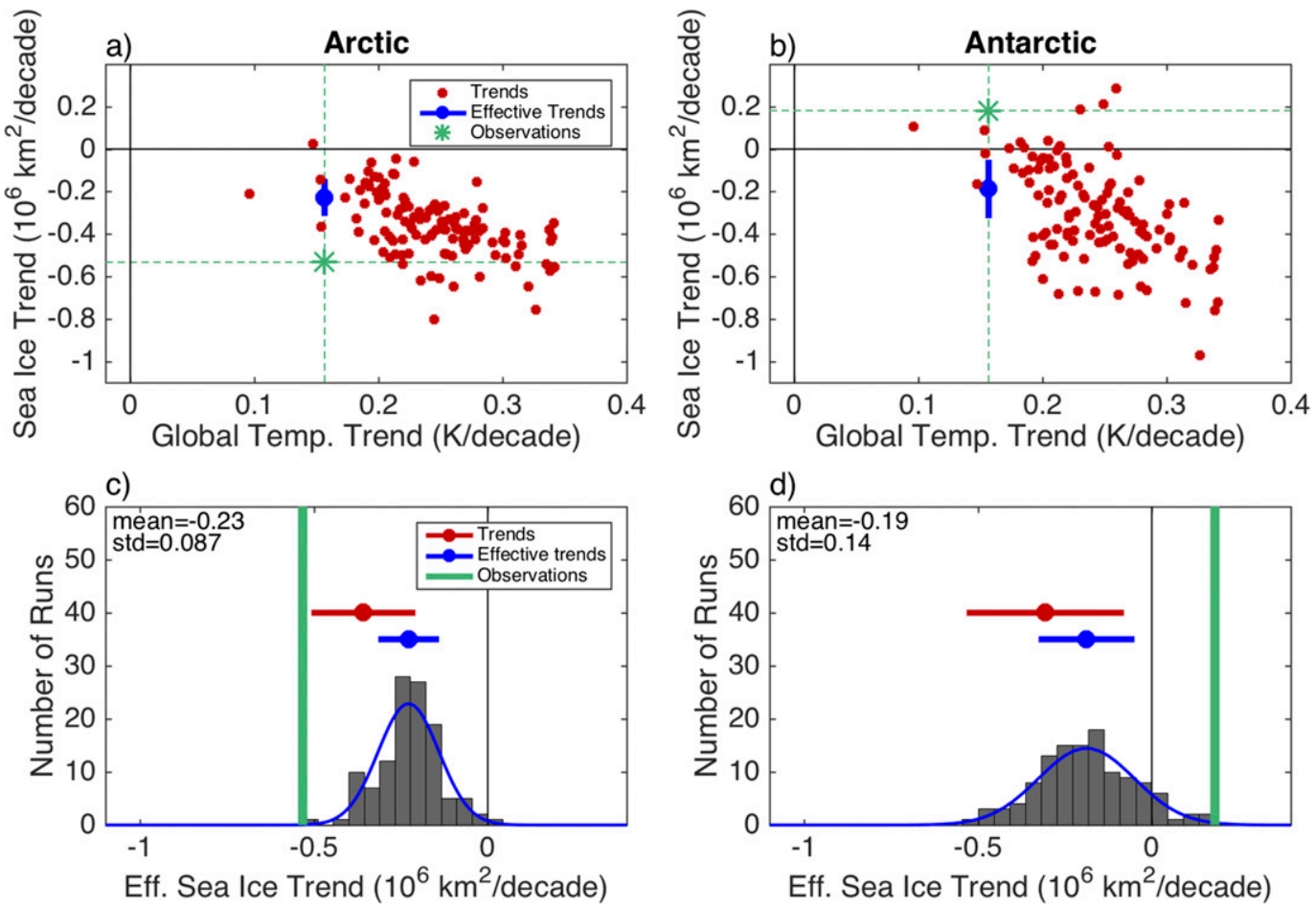

FIG. 3. As in Fig. 2, but using CMIP5 simulations instead of CESM-LE.

suggest that the underlying mechanism for the linear relationship between sea ice extent and global-mean temperature must account for the relationship being robust to changes in global-mean temperature driven by internal climate variability (cf. Winton 2011).

Next, we examine this relationship using CMIP5 simulations of 1979-2013 (Figs. 3a,b). We find here also that higher levels of global warming tend to be associated with more rapid sea ice retreat, implying that some of the intermodel differences in sea ice trends may be associated with differences in the level of simulated global warming. Comparing with observations, we find that although some of the simulations in Fig. 3a approximately match the observed sea ice retreat and others approximately match the observed level of global warming, there is a systematic bias in which none of the simulations match both observed rates. All of the simulations with Arctic sea ice trends similar to the observations have global warming rates that are approximately 1.4-2.1 times larger than the observed trend in Fig. 3a. Similarly, each simulation with a temperature trend similar to the observations underestimates the Arctic sea ice retreat by at least $30 \%$. By contrast, runs with approximately accurate levels of global warming tend to land closer to the observed Antarctic sea ice trend, although they still tend to simulate Antarctic sea ice retreat rather than the observed expansion (Fig. 3b).
Note that the relationship between sea ice trends and global-mean surface temperature trends is less correlated in the CMIP5 simulations (correlations of -0.56 and -0.54 in Figs. $3 a$ and $3 b$, respectively) than in the CESM-LE simulations (correlations of -0.73 and -0.81 in Figs. $2 a$ and $2 b$, respectively). This is consistent with the previous finding that the sea ice sensitivity to global warming remains relatively constant within a single model but can differ substantially from one model to another (Winton 2011). On the other hand, however, sea ice and global temperature are typically less correlated under internal variability than under greenhouse-driven warming (Winton 2011), which could be expected to cause simulations that differ only due to internal variability (Fig. 2) to have a less correlated relationship than simulations with different levels of greenhouse-driven warming (Fig. 3). The results of Figs. 3a,b and 2a,b suggest that the former effect is the dominant factor here, and that the low correlation among the CMIP5 simulations (Fig. 3) is largely due to intermodel differences in the sea ice sensitivity.

\section{Effective sea ice trend}

Motivated by the above result that biases in global-mean surface air temperature trends are related to both Arctic and Antarctic sea ice trends in these simulations, we consider a simple method to account for biases in the level 
of simulated global warming. This method leverages the approximately linear relationship between sea ice extent and global-mean surface temperature (Fig. S1), and it allows us to approximately estimate the distribution of sea ice trends that the models would produce if they simulated a level of global warming during 1979-2013 that matched the observations. That is, we examine how the results presented in section 2 are affected by the biases presented in section 3 .

Using the approximation that the ratio between trends in sea ice extent and trends in global temperature in each simulation does not depend on the level of global warming (which would hold if the relationship between sea ice extent and global-mean temperature were perfectly linear, i.e., if the sea ice sensitivity were constant), we can scale the sea ice trend in each simulation to account for the bias in global warming:

$$
\left(\frac{d I}{d t}\right)_{\mathrm{eff}} \equiv\left(\frac{d I}{d t} / \frac{d T}{d t}\right)_{\mathrm{sim}}\left(\frac{d T}{d t}\right)_{\mathrm{obs}} .
$$

We define the term on the left-hand side as the "effective sea ice trend," which is computed for 1979-2013 in each simulation. The effective sea ice trend is meant to approximate what the value of the simulated sea ice trend would have been if the model had accurately captured the observed level of global warming. The quotient on the right-hand side is the simulated change in sea ice extent per degree of global warming (measured in $\mathrm{km}^{2} \mathrm{~K}^{-1}$ ), which is a measure of the simulated sea ice sensitivity based on the ratio of simulated temporal trends (Winton 2011). The sea ice sensitivity is then scaled by the observed global-mean surface temperature trend, which is the final term on the right-hand side.

This method can be visualized by drawing a line from the origin through each point in Figs. 2a,b and 3a,b. The slope of this line is equivalent to the sea ice sensitivity, and the $y$ coordinate of the point where this line intersects the vertical dashed line (indicating the observed temperature trend) is equivalent to the effective sea ice trend. The spread in effective sea ice trends in each ensemble is shown by the vertical blue error bars in Figs. 2a,b and 3a,b, which indicate that the effective sea ice trends in the full ensemble are similar to the unadjusted sea ice trends in the subset of runs that have global temperature trends similar to the observations. This is consistent with the assumption of a linear relationship between sea ice area and global temperature and hence provides an approximate validation of this method.

The distributions of effective sea ice trends are plotted in Figs. 2c, 2d, 3c, and 3d. Using the effective sea ice trend causes the results presented in section 2 to change substantially (cf. red and blue confidence intervals in Figs. 2c,d and 3c,d). First, the CMIP5 ensemble-mean effective sea ice retreat is slower in each hemisphere than the unadjusted sea ice trend by more than $35 \%$. Second, the CMIP5 effective sea ice trend distribution is narrower than the distribution of unadjusted sea ice trends, implying that there is a smaller range of sea ice trends that can arise due to internal variability when constrained to match the observed level of recent global warming: the standard deviation of each distribution decreases by approximately $40 \%$ (Figs. 3c,d). Note that this may be partially related to the entire distribution being scaled by a constant value. As a result, we find that none of the 118 CMIP5 simulations has an Arctic effective sea ice retreat as fast as the observations. Similarly, none of the 118 CMIP5 simulations has an Antarctic effective sea ice expansion as large at the observations (Table 1).

Fitting a Gaussian to the distributions to approximately estimate values in the tails beyond what is populated by the 118 members, we find that the percentage of runs in the Gaussian distribution that have Arctic sea ice retreat as fast as the observations drops from $12 \%$ (Fig. 1e) to $0.02 \%$ (Fig. 3c). In the Antarctic, biases in the level of global warming appear to have a somewhat smaller effect. Although the center of the Antarctic distribution moves closer to the observed value, the width of the distribution decreases sufficiently to cause the percentage of runs in the distribution that have Antarctic sea ice expansion as large as the observations to drop from $1.6 \%$ (Fig. 1f) to $0.37 \%$ (Fig. $3 \mathrm{~d}$ ). Note that these results are qualitatively consistent with the sea ice sensitivities reported by Purich et al. (2016) and Stroeve and Notz (2016).

It is noteworthy that the discrepancies between the models and observations have similar magnitudes in the Antarctic as the Arctic when using effective sea ice trends (Figs. 3c,d), which is in contrast to the analysis of unadjusted sea ice trends, where the bias was larger in the Antarctic (Figs. 1e,f). Note that a similar finding was reported for the sea ice sensitivity in CMIP3 (Eisenman et al. 2011). This may be of interest, for example, because the different levels of consistency between the observed and modeled sea ice trends in the two hemispheres in CMIP5 contributed to the consensus view that there is low confidence in the scientific understanding of the observed Antarctic sea ice trend and high confidence in the scientific understanding of the observed Arctic sea ice trend (IPCC 2013). Overall, the results of this section imply that the possibility that internal variability alone could explain the difference between the observed and modeled sea ice trends in either hemisphere decreases substantially after accounting for biases in the level of global warming.

\section{Pseudo-ensemble from longer time period}

Next, we explore an alternative method to estimate the distribution of sea ice trends that the CMIP5 models 

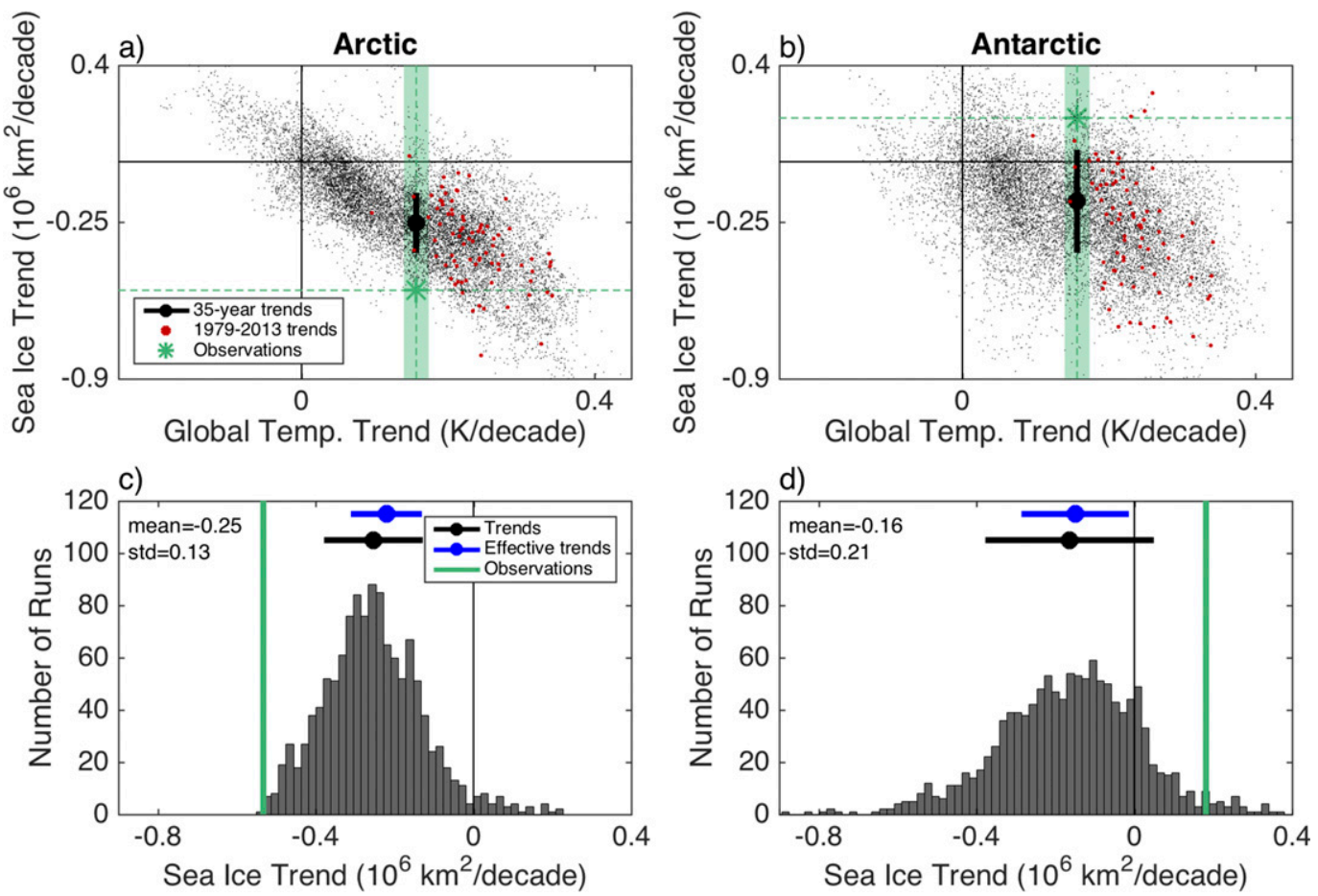

FIG. 4. Scatter of observed and simulated annual-mean (a) Arctic and (b) Antarctic sea ice trends vs the globalmean surface temperature trends from all overlapping 35-yr periods in 73 CMIP5 simulations of 1900-2100 (13 354 points in total); the 1979-2013 trends are indicated in red (as in Figs. 3a,b). Green dashed horizontal and vertical lines represent the observed trends. Global-mean surface temperature trends that are within one standard deviation of the observed trend are highlighted in green. (c),(d) Sea ice trends from periods that fall within the highlighted regions are shown in histograms with the observed trend indicated by a thick green line. Standard deviations of this distribution (black error bars) and the distribution of 1979-2013 effective sea ice trends (blue error bars, as in Figs. $3 \mathrm{c}, \mathrm{d})$ are also shown. The mean and standard deviation of the trends that fall within the highlighted regions are repeated for comparison in the top panel (black vertical error bars).

would simulate if each run had the observed level of global warming during 1979-2013. Here we assume that the relationship between global warming and sea ice changes is the same for all 35-yr periods (which would hold if the relationship between sea ice extent and globalmean temperature were perfectly linear, i.e., if the sea ice sensitivity were constant). We therefore examine the trends during each overlapping 35 -yr period in each of the CMIP5 simulations of 1900-2100. There are a total of 13354 overlapping 35-yr periods (some CMIP5 runs were excluded because data were not available for the entire 1900-2100 period; see appendix A for details). Figures 4a and $4 \mathrm{~b}$ show a scatter of these 13354 trends in annualmean global-mean surface air temperature and sea ice extent. The trends during 1979-2013 are shown in red, illustrating the qualitatively similar relationship between trends in sea ice and global temperature during this period and other 35 -yr periods. That is, we find that higher levels of global warming are associated with faster sea ice retreat, even over this extended range of trends in global-mean surface temperature.
To validate whether this method can provide a meaningful approximation to the ensemble of 1979-2013 simulation results, we consider the distribution of sea ice trends during all 35-yr periods that have levels of global warming similar to the simulated 1979-2013 distribution (i.e., similar to Fig. 1d). Specifically, we select the 3923 periods during 1900-2100 that have temperature trends within one standard deviation of the 1979-2013 ensemble mean (points that fall within the red shaded region in Figs. S3a,b), and we examine the histogram of the corresponding sea ice trends (Figs. S3c,d). We find that the distribution of 3923 trends in Figs. S3c and S3d does approximately match the mean and standard deviation of the smaller distribution of 118 simulated sea ice trends during 1979-2013 (Figs. 1e,f): the red and black error bars in Figs. S3c and S3d are approximately aligned. This implies that this method allows us to build a far larger "pseudo-ensemble" of sea ice trends by harvesting time periods with similar levels of global warming from the 200-yr simulations.

Next, we create a pseudo-ensemble of time periods in the simulations that have global warming trends similar 
to the 1979-2013 observed value. This provides an approximation of the spread of simulated sea ice trends that would coincide with the observed level of global warming. Green shading in Figs. $4 \mathrm{a}$ and $4 \mathrm{~b}$ indicates $35-\mathrm{yr}$ global warming trends that are within the $68 \%$ linear regression confidence interval of the observed trend (using the method in section $\mathrm{b}$ of appendix B to account for autocorrelation). The pseudo-ensemble plotted in Figs. 4c and $4 \mathrm{~d}$ comprises the distribution of 123235 -yr periods that fall within this green shaded region. This approximates the ensemble of periods whose distribution of global warming trends is consistent with the observed trend.

Only $1(0.08 \%)$ of the 1232 sea ice trends from this pseudo-ensemble has an Arctic sea ice retreat that is as large as the observations (Table 1). Therefore, this analysis of years 1900-2100 in the simulations yields a similar result to the analysis in section 5 that used the 1979-2013 effective sea ice trends. Consistent with this, the pseudoensemble distribution (Fig. 4c) has a mean that is similar to the distribution of effective sea ice trends (Fig. 3c), although the standard deviations of the two distributions are somewhat different. This can be seen by comparing the black and blue error bars in Fig. 4c.

Note that increasing the range of global warming trends included in the pseudo-ensemble (i.e., widening the green shaded region in Fig. 4a) does not substantially influence these results. Specifically, when we use the $95 \%$ autocorrelation-corrected linear regression confidence interval of the observed trend rather than the $68 \%$ confidence interval, we find that $6(0.24 \%)$ of the 2532 periods in the pseudo-ensemble have Arctic sea ice retreat as fast as the observations. See section c of appendix B for an alternative approach to generating a pseudo-ensemble that accurately captures the target distribution.

Similar to the comparison in section 4 between the effective sea ice trend distribution and the unadjusted sea ice trend distribution, we next compare the pseudoensemble associated with the observed 1979-2013 level of global warming (Fig. 4c) with the pseudo-ensemble associated with the ensemble of simulated 1979-2013 levels of global warming (Fig. S3c). First, whereas $9.0 \%$ of the Arctic sea ice trends in Fig. S3c are at least as negative as the observed value, this value drops to $0.08 \%$ in Fig. 4c. Second, the mean Arctic sea ice trend in the pseudo-ensemble associated with the simulated level of 1979-2013 global warming (Fig. S3c) is approximately $25 \%$ larger than in the pseudo-ensemble associated with the observed level of global warming (Fig. 4c). These results are approximately similar to the effective sea ice trend results in section 4 .

Turning to the Antarctic, we find some qualitative similarities with the Arctic results. First, the mean of the Antarctic sea ice trends in the pseudo-ensemble associated with the observed level of global warming is similar to the ensemble mean of Antarctic effective sea ice trends, although the standard deviations of the two distributions are somewhat different (error bars in Fig. 4d). Second, the mean Antarctic sea ice trend in the pseudo-ensemble associated with the observed level of global warming (Fig. 4 d) is about $30 \%$ smaller than in the pseudo-ensemble associated with the CMIP5 simulated level of global warming (Fig. S3d). A notable differences compared with the Arctic results is that $3.7 \%$ of the periods in the pseudo-ensemble associated with the observed level of global warming have Antarctic sea ice expansion as large as the observations (Table 1, Fig. 4d), compared to $1.6 \%$ of the periods in the pseudoensemble associated with the CMIP5 simulated level of warming (Fig. S3d). This is in contrast with the effective sea ice trend results in section 5 , where the fraction of Antarctic sea ice trends as positive as the observations was found to be smaller for the effective sea ice trend than for the unadjusted sea ice trend. The reason for this discrepancy between the pseudoensemble result here and the effective trend result in section 5 may be related to issues with the Antarctic sea ice sensitivity varying during the 1900-2100 period. In Fig. S1b, the Antarctic sea ice sensitivity in CESM-LE can be seen to be larger during 1900-2000 (top left part of plot: small warming leads to large sea ice retreat) than during 2001-2100 (remainder of plot: further warming leads to more gradual sea ice retreat). This may be associated with the Antarctic sea ice sensitivity being influenced by ozone forcing or other local processes that do not scale with greenhouse forcing during 1900-2100. The sea ice sensitivity in CESM-LE is more constant in the Arctic during 1900-2100 (Fig. S1a). This may cause the pseudo-ensemble approach, which assumes constant sea ice sensitivity during 1900-2100, to be less accurate in the Antarctic than the Arctic (cf. Fig. S2).

\section{Discussion}

The relationship between the global-mean surface air temperature trend and both the Arctic and Antarctic sea ice trends in these simulations implies that the models do not capture the hemispheric asymmetry of the observed sea ice trends during 1979-2013. This is illustrated in Fig. 5a, which indicates a substantial systematic bias in the CESM-LE simulations compared with observations. The sea ice trend is more accurately simulated in one hemisphere only at the cost of accuracy in the other. This is closely related to the temperature trend in each realization of internal variability (colors of points in Fig. 5a). Realizations that warm most rapidly compared to observations (red and orange points) tend to have more accurate 

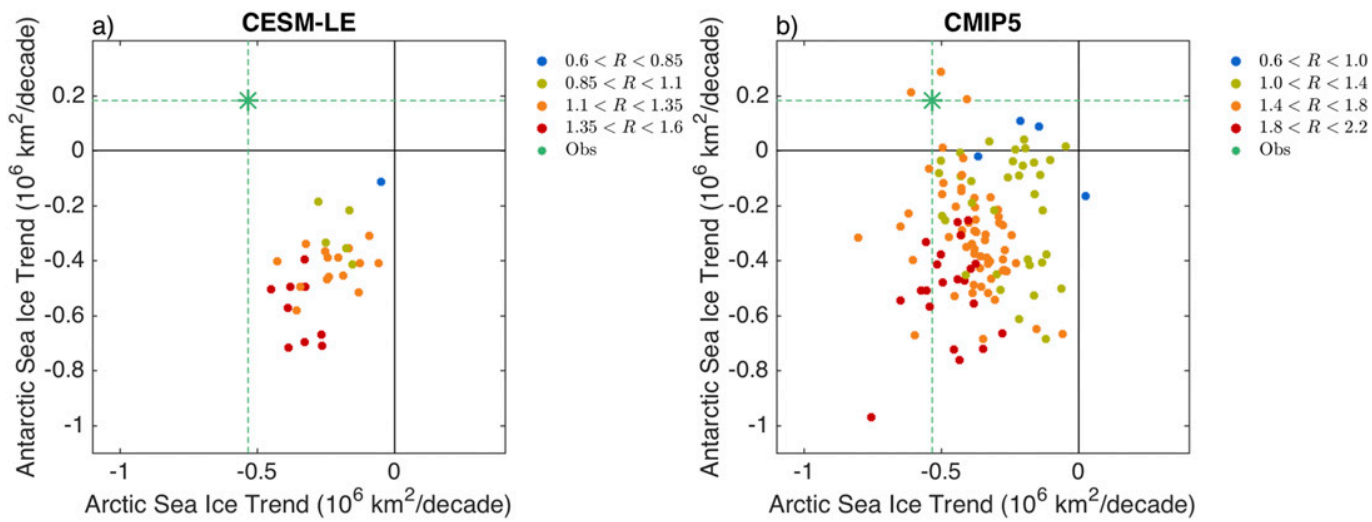

$1.0<R<1.4$

- $1.4<R<1.8$

- $1.8<R<2.2$

- Obs

FIG. 5. Simulated annual-mean Antarctic sea ice trend vs Arctic sea ice trend in each run from the (a) CESM-LE and (b) CMIP5 ensembles. The observed trends are indicated by green dashed horizontal and vertical lines. The color of each point indicates the ratio $R$ between the simulated and observed values of the annual-mean globalmean surface temperature trend in each simulation.

Arctic sea ice trends but greater biases toward Antarctic sea ice retreat rather than expansion. The reverse also appears to be true (blue and yellow points), although there are far fewer simulations in CESM-LE that underestimate global warming trends. We repeat this analysis using CMIP5 in Fig. 5b and find a similar result, although there is more spread, as expected from the comparison of Figs. 2a,b with Figs. 3a,b. Note that there are three simulations (from IPSL-CM5A-LR, MPI-ESM-MR, and BCCCSM1.1) that simulate sea ice retreat that is similar to the observations in both hemispheres, but they each overestimate the level of global warming by at least $40 \%$.

The analyses in sections 5 and 6 rely on the approximation that the relationship between simulated sea ice extent and global-mean surface air temperature is linear. The accuracy of this approximation for climate model simulations is demonstrated in Fig. S1. In the Arctic, simulated sea ice extent is highly correlated with global-mean temperature (left column of Fig. S1). This close relationship is consistent with the previous finding that the Arctic sea ice sensitivity in a given model does not depend on the forcing scenario (Winton 2011). While the Antarctic sea ice extent is not as highly correlated with global-mean temperature in many of the models (right column of Fig. S1), the distributions in Figs. 2b, 3b, and $4 b$ suggest that the correlations between Antarctic sea ice extent and global-mean surface air temperature may be sufficiently large that the relationship between the trends of these two values during $35-\mathrm{yr}$ periods are directly related, causing simulated Antarctic sea ice expansion to occur more often during periods with less global warming than observed during 1979-2013.

We examine the extent to which internal climate variability weakens the relationship between sea ice extent and global-mean surface temperature over short time scales by evaluating the distribution of Arctic and Antarctic sea ice sensitivity in 30 CESM-LE simulations of 2006-2100 (Figs. S5e,f). This time period is chosen to avoid issues with the dependence of Antarctic sea ice sensitivity on the time period, as discussed in section 6 above (Fig. S1b). We then compute the sea ice sensitivity of each overlapping 55-yr period (Figs. S5c,d) and 35-yr period (Figs. S5a,b). The greater widths of the latter distributions indicate the extent to which internal variability influences this relationship over shorter time periods. Figure S5 indicates that even for 35-yr time periods, the distributions of sea ice sensitivities in both hemispheres remain relatively narrow compared with the distance from the origin to the center of each distribution; that is, the fractional spreads remain relatively small.

Note that by approximating that sea ice extent varies linearly with global-mean temperature (Fig. S1) in the effective sea ice trend and pseudo-ensemble analyses (sections 4 and 5), we approximate here that the sea ice sensitivity takes the same value in a given simulation whether the global warming occurs due to rising greenhouse forcing or internal variability (i.e., that the sea ice sensitivity is constant). However, it has previously been shown in a climate model that the magnitude of the Arctic sea ice sensitivity is somewhat larger in a control simulation than in a forced warming simulation (Winton 2011). That is, it was found that there was more sea ice retreat under global warming caused by internal variability than under the same level of global warming caused by rising greenhouse forcing. This effect appears to also occur in the analysis presented here for both the Arctic and the Antarctic. In CESM-LE, all of the simulations of 1979-2013 have the same global warming due to greenhouse forcing since they are all 
from the same model with the same forcing scenario, but the temperature trends differ among the simulations due to internal variability. Hence a CESM-LE simulation with a larger temperature trend has more warming due to internal variability, and thus it should show a sea ice sensitivity with a larger magnitude. Indeed, points in Figs. $2 \mathrm{a}$ and $2 \mathrm{~b}$ that are farther to the right (i.e., runs with larger global temperature trends) tend to have a ratio of the sea ice trend to the global temperature trend with a larger magnitude (i.e., the magnitude of the sea ice sensitivity is larger). Similar arguments imply that smaller temperature trends have a smaller magnitude of this ratio. This also occurs to a lesser extent in CMIP5 (Figs. 3a,b), where the global warming due to greenhouse forcing varies among the runs. Hence this effect may explain why the scatterplots in Figs. 2a, 2b, 3a, and $3 \mathrm{~b}$ do not appear to linearly extrapolate through the origin.

The central results of this study are relevant to previous studies that used control simulations with constant forcing to determine whether the observed Arctic and Antarctic sea ice trends could arise due to internal variability alone (Kay et al. 2011; Polvani and Smith 2013; Mahlstein et al. 2013; Jones et al. 2016). For example, Polvani and Smith (2013) found that 1979-2005 Antarctic sea ice trends that are up to 3 times as large as the observed trend can naturally emerge in control simulations. They suggest that this implies that the internal variability of this system is large enough to overwhelm the forced global warming signal, similar to arguments made by Mahlstein et al. (2013). However, the results presented here imply that periods in control simulations with expanding Antarctic sea ice are likely to have global warming trends that are substantially below the 1979-2013 observed trend. Therefore, these results imply that when simulated global warming trends are not considered, neither the center nor the width of the distribution of sea ice trends in a control simulation should be expected to accurately reflect the range of possible sea ice trends that can emerge in climate models under the observed level of global warming.

We have examined the sensitivity of the results presented here to adjustments in various details of the analysis, which is discussed in appendix B. In appendix B, section a, we evaluate the influence of using sea ice area in the models and observations, rather than sea ice extent as used in the main text. In the appendix B, section $\mathrm{b}$, we repeat the analyses from sections 2 and 4 using a framework in which each run is treated as a single realization from a unique ensemble, following Stroeve et al. (2012) and Santer et al. (2008); this is in contrast to the analysis in the main text, which treated all runs as realizations from a single ensemble. In appendix $\mathrm{B}$, section $\mathrm{c}$, we carry out an alternative pseudo-ensemble approach that is more complicated than that used in section 6 but may more accurately capture the target distribution. In appendix B, section d, we repeat the effective sea ice trend analysis (section 4) with the sea ice sensitivity in Eq. (1) computed using a total least squares regression, rather than the ratio of ice and temperature temporal trends. In appendix B, section e, we repeat the effective trend analysis (section 4) and the pseudo-ensemble analysis (section 5) using the Hadley Centre Climatic Research Unit version 4 (HadCRUT4) dataset (Morice et al. 2012) for the globalmean surface temperature, rather than the GISTEMP dataset. Consistent with the central results of this study, in each case we find that the possibility that internal variability alone could explain the difference between simulated and observed sea ice trends in either hemisphere becomes exceedingly small after we account for biases in the level of global warming.

The results presented here stem from the point that the observed relationship between sea ice extent and global-mean surface temperature (i.e., the observed sea ice sensitivity) is markedly different in each hemisphere from that simulated by climate models. It should be emphasized that the physical processes that determine the ice sensitivity are not well understood. Therefore, this bias may be related to issues in the atmosphere, ocean, or sea ice model components that are connected to the simulated sea ice changes or to the simulated level of global warming. For example, several studies have identified model biases related to global warming trends (e.g., IPCC 2013; Kosaka and Xie 2013) and local processes that influence sea ice (Rampal et al. 2011; Jahn et al. 2012; Mahlstein and Knutti 2012; Bintanja et al. 2013; Mahlstein et al. 2013; Zunz et al. 2013; Uotila et al. 2014; Haumann et al. 2014; Purich et al. 2016; Jones et al. 2016). Additional studies have suggested that polar teleconnections may also have an important influence on sea ice trends in each hemisphere (Meehl et al. 2016; Screen and Francis 2016). Furthermore, errors in the observations could plausibly contribute to the discrepancy between observed and modeled sea ice sensitivity. For example, several studies have suggested that poorly sampled observations around the poles and in parts of Africa may help explain differences between observed and modeled global-mean surface temperature trends (Cowtan and Way 2014; Richardson et al. 2016; Karl et al. 2015). Similarly, recent studies have highlighted uncertainties in the observed multidecadal Antarctic sea ice extent trend due to changes in data sources (Screen et al. 2011; Eisenman et al. 2014). Lastly, we find that the observations show a 
correlation between sea ice extent and global-mean surface temperature that is similar to the models in the Arctic but not in the Antarctic (Fig. S6). This suggests that the discrepancy between the models and the observations in the Antarctic could be related to the models simulating an unrealistically tight relationship between Antarctic sea ice extent and global temperatures.

\section{Conclusions}

In each hemisphere, the observed 1979-2013 trend in sea ice extent falls at least marginally within the distribution of the CMIP5 simulations (Figs. 1b,c,e,f). Consistent with this, a number of previous studies have suggested that internal climate variability could explain the difference between the observed sea ice trend and the ensemble-mean simulated trend in each hemisphere (Kay et al. 2011; Stroeve et al. 2012; Polvani and Smith 2013; Mahlstein et al. 2013; Turner et al. 2013; Swart and Fyfe 2013; Zunz et al. 2013; IPCC 2013; Fan et al. 2014; Notz 2014; Gagné et al. 2015; Goosse and Zunz 2014; Swart et al. 2015; Purich et al. 2016; Jones et al. 2016).

The results presented here suggest that this viewpoint breaks down when we account for biases in simulated 1979-2013 global-mean surface temperature trends. We find that simulated Arctic sea ice retreat is accurate only in runs that have far too much global warming (Figs. 2a, 3a, 4a). This suggests that the models may be getting the right Arctic sea ice retreat for the wrong reasons. Similarly, simulated periods with accurate Antarctic sea ice trends tend to have too little global warming, although these results are more equivocal (Figs. 2b, 3b, 4b). Relatedly, the simulations do not capture the observed asymmetry between Arctic and Antarctic sea ice trends (Fig. 5).

We quantify how this bias influences the level of agreement between models and observations (Fig. 1) by estimating what the simulated sea ice trend in each hemisphere would be in runs that matched the observed level of global warming (Table 1). This analysis relies on the approximately linear relationship between sea ice extent and global-mean surface temperature in the simulations (Fig. S1), which allows us to scale the results from simulations with varied levels of global warming (Figs. $2 \mathrm{c}, \mathrm{d}$ and $3 \mathrm{c}, \mathrm{d}$ ) or use simulations from different time periods (Figs. 4c,d). These results suggest that the difference between observed and modeled sea ice trends in each hemisphere cannot be attributed to simulated internal climate variability alone. This implies systematic errors in the Arctic and Antarctic sea ice changes simulated with current climate models, or possibly errors in the observations.
Acknowledgments. Without implying their endorsement, we are grateful to Sarah Gille, Art Miller, Paul Kushner, Neil Tandon, Frédéric Laliberté, Till Wagner, and John Fyfe for helpful comments and discussions. This work was supported a National Science Foundation (NSF) Graduate Research Fellowship and NSF Grants ARC-1107795 and OCE-1357078. We acknowledge the World Climate Research Programme's Working Group on Coupled Modelling, which is responsible for CMIP5, and we thank the climate modeling groups (listed in Table S1 in the online supplemental material) for producing and making available their model output. We also acknowledge the CESM Large Ensemble Community Project and supercomputing resources provided by NSF/ CISL/Yellowstone. Processed CMIP5 data used in this study are available at http://eisenman.ucsd.edu/code.html.

\section{APPENDIX A}

\section{Methods}

Here further details are given regarding the observations and the processing of the CMIP5 model output.

For the observed sea ice extent and sea ice area, we use monthly-mean data from the National Snow and Ice Data Center Sea Ice Index (Fetterer et al. 2002), which uses the NASA Team algorithm to estimate sea ice concentration from satellite passive microwave measurements. We analyze years 1979-2013, since this was the period available at the time of analysis. We fill missing monthly values by interpolating between the same months in the previous and following years, and we then take annual averages. For the observed annualmean global-mean surface temperature data, we use the Goddard Institute for Space Sciences Surface Temperature Analysis (GISTEMP) (Hansen et al. 2010).

We analyze 118 simulations of years 1979-2013 from 40 CMIP5 models, using the Historical (1850-2005) and RCP4.5 (2006-2100) experiments; note that the choice of RCP scenario has minimal influence during 2006-13. The models simulate surface air temperature at each horizontal atmospheric grid point, and sea ice concentration is simulated on the ocean grid in many of the models. Therefore, the areas of the cells in both grids are often needed to compute the total Arctic and Antarctic sea ice areas as well as the global-mean temperature. The following models did not have grid cell areas reported in the CMIP5 archive: CanCM4 (surface air temperature), MPI-ESM-LR (surface air temperature), and FIO-ESM (surface air temperature and sea ice). In these cases, grid cell areas were estimated from the reported locations of grid cell corners using the Haversine formula (note that this method requires a regular grid). 
Simulations were not analyzed in this study when surface air temperature output was not available during all of 1979-2013, sea ice output was not available during all of 1979-2013, dates reported in the file did not match the filename in the CMIP5 archive, or irregular grids were used but grid cell areas were not provided. The following runs each had at least one of these issues and hence were excluded: EC-EARTH runs 1, 3-6, and 10; FIO-ESM run 2; MIROC-ESM-CHEM run 2; CESM1CAM5-1-FV2 runs 1-4; GFDL-CM3 runs 2-5; GFDLCMP2p1 runs 1-10; all runs from BCC-CSM1-1-M; and all runs from INMCM. GFDL-ESM2G run 1 is also excluded because the Antarctic sea ice extent gradually decreases and then increases during 1979-2013, leading to a highly autocorrelated time series of linear regression residuals with less than 2 effective degrees of freedom, which causes the standard error in the analysis in appendix B, section b to be complex [cf. Eq. (4) in Santer et al. 2008]. Additional simulations were excluded from the analysis in Fig. 4 and Figs. S1e,f because data were not available for the entire 19002100 period.

\section{APPENDIX B}

\section{Robustness to Changes in Methods}

\section{a. Using ice area instead of ice extent}

The results presented in the main text use sea ice extent as a measure of the sea ice cover. Here we briefly summarize the effect of instead using sea ice area in the models and observations. First, considering the Gaussian distribution of sea ice trends (as in Figs. 1e,f), we find that $22 \%$ of the simulations would have Arctic sea ice retreat that is as fast as the observations, and $1.5 \%$ would have Antarctic sea ice expansion at least as large as the observations, similar to the values of $12 \%$ and $1.6 \%$, respectively, that we found for ice extent. When we use the Gaussian distribution of Arctic and Antarctic effective sea ice trends (as in Figs. 3c,d), these values drop to $0.15 \%$ and $0.28 \%$ (similar to $0.02 \%$ and $0.37 \%$ for ice extent), respectively. Lastly, of the 1232 overlapping 35 -yr periods that have global warming trends that are similar to the 1979-2013 observations (as in Figs. 4c,d), $1.3 \%$ of the periods have Arctic sea ice trends as negative as the observed value, and $3.1 \%$ of the periods have Antarctic sea ice trends as positive as the observed value (similar to $0.08 \%$ and $3.7 \%$, respectively, for ice extent).

\section{b. Paired trends tests}

In this section, we consider an alternative framework for the analysis in the main text: rather than treat each CMIP5 simulation as a realization from a single model, here we treat each simulation as a realization from a separate model. Following previous studies (Santer et al. 2008; Stroeve et al. 2012), we determine if each simulated trend is statistically different from the observed trend at the $95 \%$ confidence level by using Welch's $t$-test statistic:

$$
d=\frac{\beta_{m}-\beta_{o}}{\sqrt{\sigma_{m}^{2}+\sigma_{o}^{2}}} .
$$

Here $\beta_{m}$ and $\beta_{o}$ are the modeled and observed trends, respectively, and $\sigma_{m}$ and $\sigma_{o}$ are the associated standard errors, which are adjusted for autocorrelation following Santer et al. (2008). A value of $|d|>1.96$ is equivalent to zero falling outside of the $95 \%$ confidence interval of a Gaussian distribution with a mean of $\beta_{m}-\beta_{o}$ and a standard deviation of $\sqrt{\sigma_{m}^{2}+\sigma_{o}^{2}}$. In Figs. S7a and S7b, $\beta_{m}$ and $\beta_{o}$ are the observed and modeled sea ice trends, which are represented by a series of solid black dots (one for each simulation). The standard errors $\sigma_{m}$ and $\sigma_{o}$ are also shown. In Figs. S7c and S7d, $\beta_{m}$ is the effective sea ice trend and $\sigma_{m}$ is determined using error propagation:

$$
\sigma_{m}=\sqrt{\left(\frac{\beta_{m}}{\beta_{I_{t}}} \sigma_{I_{t}}\right)^{2}+\left(\frac{\beta_{m}}{\beta_{T_{t}}} \sigma_{T_{t}}\right)^{2}+\left(\frac{\beta_{m}}{\beta_{T_{t}^{o}}} \sigma_{T_{t}^{o}}\right)^{2}}
$$

where $\beta_{I_{t}}, \beta_{T_{t}}$, and $\beta_{T_{t}^{o}}$ are the trends in simulated sea ice, simulated global-mean surface temperature, and observed global-mean surface temperature, and $\sigma_{I_{t}}, \sigma_{T_{t}}$, and $\sigma_{I_{t}}$ are the associated standard errors.

We find that of the 118 simulations, $33 \%$ simulate sea ice trends that are statistically different from the observations at the $95 \%$ confidence level in the Arctic, and $80 \%$ in the Antarctic. On the other hand, $81 \%$ and $84 \%$ of the simulations have Arctic and Antarctic effective sea ice trends that are different from the observations at the $95 \%$ level, respectively.

\section{c. Using scaled histograms in pseudo-ensemble analysis}

In this section, we repeat the calculation in section 5 (Fig. 4) using a somewhat more precise but less straightforward approach that involves a weighting function rather than simply selecting the runs that fall within the shaded region. We begin with the distribution of 13354 overlapping 35-yr temperature trends during 1900-2100 as well as a Gaussian distribution centered on the observed temperature trend with a width equal to the $68 \%$ linear regression confidence interval (which is adjusted for autocorrelation, as in the previous subsection). Next, we assign each $35-\mathrm{yr}$ period a weight equal to the height of the Gaussian at the center of the 
histogram bin where the 35-yr period falls divided by the number of runs in the histogram bin.

These weights scale the distribution of temperature trends during 1900-2100 to match a distribution consistent with the observed 1979-2013 temperature trend. Next, we create a histogram of sea ice trends with each 35-yr period multiplied by its weight. Hence this approach asks what range of ice extent trends is consistent with the observed temperature trend under the assumptions described in section 5. We find that the resulting distribution is approximately equivalent to the result of the simpler approach in section 5 (Figs. $4 \mathrm{c}, \mathrm{d}$ ): $0.32 \%$ of the 35 -yr periods have Arctic sea ice trends are at least as negative as the observed value, and 5.2\% have Antarctic sea ice trends at least as positive as the observed value, compared with $0.08 \%$ and $3.7 \%$, respectively, reported in section 5 .

\section{d. Using total least squares to compute sea ice sensitivity}

Winton (2011) found that computing the sea ice sensitivity using a total least squares (TLS) regression between sea ice extent and global-mean surface air temperature leads to a slightly more accurate estimate than the ratio of ice and temperature temporal trends as in Eq. (1). We find that replacing the ratio of trends in Eq. (1) with a TLS regression between ice extent and global-mean surface air temperature yields similar results: using Gaussian fits to the distributions, we find that the probability that the observations would land this far below or above the TLS effective sea ice trend ensemble mean is $0.02 \%$ and $0.08 \%$ in the Arctic and Antarctic, respectively (similar to $0.02 \%$ and $0.37 \%$, respectively, computed using the trend ratio).

\section{e. Using HadCRUT4 instead of GISTEMP}

The results presented in the main text use the GISTEMP dataset for the observed annual-mean globalmean surface temperature. Here we briefly summarize the effect of instead using the HadCRUT4 dataset (Morice et al. 2012). This causes the 1979-2013 temperature trend to increase from $0.157 \mathrm{Kdecade}^{-1}$ (GISTEMP) to $0.159 \mathrm{~K} \mathrm{decade}^{-1}$ (HadCRUT4). Considering the Gaussian distribution of effective sea ice trends (as in Figs. 3c,d), we find that this leads to $0.03 \%$ (HadCRUT4) instead of $0.02 \%$ (GISTEMP) of the simulations having Arctic sea ice retreat that is as fast as the observations, and $0.39 \%$ (HadCRU4) instead of 0.37\% (GISTEMP) having Antarctic sea ice expansion as fast as the observations. Considering the 1232 overlapping 35-yr periods that have global warming trends similar to the 1979-2013 observations (as in Figs. 4c,d), we find that this causes $0.08 \%$ (HadCRUT as in GISTEMP) of the periods to have Arctic sea ice trends as negative as observed, and $3.5 \%$ (HadCRUT) instead of $3.7 \%$
(GISTEMP) of the periods to have Antarctic sea ice trends as positive as observed. In summary, switching from GISTEMP to HadCRUT4 has little effect on the main results presented here.

\section{REFERENCES}

Armour, K. C., I. Eisenman, E. Blanchard-Wrigglesworth, K. E. McCusker, and C. M. Bitz, 2011: The reversibility of sea ice loss in a state-of-the-art climate model. Geophys. Res. Lett., 38, L16705, doi:10.1029/2011GL048739.

Bintanja, R., G. J. van Oldenborgh, S. S. Drijfhout, B. Wouters, and C. A. Katsman, 2013: Important role for ocean warming and increased ice-shelf melt in Antarctic sea-ice expansion. Nat. Geosci., 6, 376-379, doi:10.1038/ngeo1767.

Comiso, J. C., and F. Nishio, 2008: Trends in the sea ice cover using enhanced and compatible AMSR-E, SSM/I, and SMMR data. J. Geophys. Res., 113, C02S07, doi:10.1029/2007JC004257.

Cowtan, K., and R. G. Way, 2014: Coverage bias in the HadCRUT4 temperature series and its impact on recent temperature trends. Quart. J. Roy. Meteor. Soc., 140, 1935-1944, doi:10.1002/qj.2297.

Eisenman, I., T. Schneider, D. S. Battisti, and C. M. Bitz, 2011: Consistent changes in the sea ice seasonal cycle in response to global warming. J. Climate, 24, 5325-5335, doi:10.1175/2011JCLI4051.1.

, W. N. Meier, and J. R. Norris, 2014: A spurious jump in the satellite record: Has Antarctic sea ice expansion been overestimated? Cryosphere, 8, 1289-1296, doi:10.5194/tc-8-1289-2014.

Fan, T., C. Deser, and D. P. Schneider, 2014: Recent Antarctic sea ice trends in the context of Southern Ocean surface climate variations since 1950. Geophys. Res. Lett., 41, 2419-2426, doi:10.1002/2014GL059239.

Fetterer, F., K. Knowles, W. Meier, and M. Savoie, 2002 (updated 2012): Sea ice index. National Snow and Ice Data Center, accessed June 2016. [Available online at http://nsidc.org/data/ g02135.html.]

Gagné, M.-È., N. P. Gillett, and J. C. Fyfe, 2015: Observed and simulated changes in Antarctic sea ice extent over the past 50 years. Geophys. Res. Lett., 42, 90-95, doi:10.1002/2014GL062231.

Goosse, H., and V. Zunz, 2014: Decadal trends in the Antarctic sea ice extent ultimately controlled by ice-ocean feedback. Cryosphere, 8, 453-470, doi:10.5194/tc-8-453-2014.

Gregory, J. M., P. A. Stott, D. J. Cresswell, N. A. Rayner, C. Gordon, and D. M. H. Sexton, 2002: Recent and future changes in Arctic sea ice simulated by the HadCM3 AOGCM. Geophys. Res. Lett., 29, 2175, doi:10.1029/2001GL014575.

Hansen, J., R. Ruedy, M. Sato, and K. Lo, 2010: Global surface temperature change. Rev. Geophys., 48, RG4004, doi:10.1029/ 2010RG000345.

Haumann, F. A., D. Notz, and H. Schmidt, 2014: Anthropogenic influence on recent circulation-driven Antarctic sea ice changes. Geophys. Res. Lett., 41, 8429-8437, doi:10.1002/2014GL061659.

IPCC, 2007: Climate Change 2007: The Physical Science Basis. S. Solomon et al., Eds., Cambridge University Press, 996 pp.

—, 2013: Climate Change 2013: The Physical Science Basis. T. F. Stocker et al., Eds., Cambridge University Press, 1535 pp.

Jahn, A., and Coauthors, 2012: Late-twentieth-century simulation of Arctic sea ice and ocean properties in the CCSM4. J. Climate, 25, 1431-1452, doi:10.1175/JCLI-D-11-00201.1.

Jones, J. M., and Coauthors, 2016: Assessing recent trends in highlatitude Southern Hemisphere surface climate. Nat. Climate Change, 6, 917-926, doi:10.1038/nclimate3103. 
Karl, T. R., and Coauthors, 2015: Possible artifacts of data biases in the recent global surface warming hiatus. Science, $\mathbf{3 4 8}, 1469$ 1472, doi:10.1126/science.aaa5632.

Kay, J. E., M. M. Holland, and A. Jahn, 2011: Inter-annual to multi-decadal Arctic sea ice extent trends in a warming world. Geophys. Res. Lett., 38, L15708, doi:10.1029/2011GL048008.

— , and Coauthors, 2015: The Community Earth System Model (CESM) large ensemble project: A community resource for studying climate change in the presence of internal climate variability. Bull. Amer. Meteor. Soc., 96, 1333-1349, doi:10.1175/BAMS-D-13-00255.1.

Kosaka, Y., and S.-P. Xie, 2013: Recent global-warming hiatus tied to equatorial Pacific surface cooling. Nature, 501, 403-407, doi:10.1038/nature12534.

Mahlstein, I., and R. Knutti, 2012: September Arctic sea ice predicted to disappear near $2^{\circ} \mathrm{C}$ global warming above present. J. Geophys. Res., 117, D06104, doi:10.1029/2011JD016709.

—, P. R. Gent, and S. Solomon, 2013: Historical Antarctic mean sea ice area, sea ice trends, and winds in CMIP5 simulations. J. Geophys. Res. Atmos., 118, 5105-5110, doi:10.1002/ jgrd.50443.

Meehl, G. A., C. Covey, K. E. Taylor, T. Delworth, R. J. Stouffer, M. Latif, B. McAvaney, and J. F. B. Mitchell, 2007: THE WCRP CMIP3 multimodel dataset: A new era in climate change research. Bull. Amer. Meteor. Soc., 88, 1383-1394, doi:10.1175/BAMS-88-9-1383.

, J. M. Arblaster, C. M. Bitz, C. T. Y. Chung, and H. Teng, 2016: Antarctic sea-ice expansion between 2000 and 2014 driven by tropical Pacific decadal climate variability. Nat. Geosci., 9, 590-595, doi:10.1038/ngeo2751.

Morice, C. P., J. J. Kennedy, N. A. Rayner, and P. D. Jones, 2012: Quantifying uncertainties in global and regional temperature change using an ensemble of observational estimates: The HadCRUT4 data set. J. Geophys. Res., 117, D08101, doi:10.1029/2011JD017187.

Notz, D., 2014: Sea-ice extent and its trend provide limited metrics of model performance. Cryosphere, 8, 229-243, doi:10.5194/ tc-8-229-2014.

— follows anthropogenic $\mathrm{CO}_{2}$ emission. Science, 354, 747-750, doi:10.1126/science.aag2345.

Polvani, L. M., and K. L. Smith, 2013: Can natural variability explain observed Antarctic sea ice trends? New modeling evidence from CMIP5. Geophys. Res. Lett., 40, 3195-3199, doi:10.1002/grl.50578.

Purich, A., W. Cai, M. H. England, and T. Cowan, 2016: Evidence for link between modelled trends in Antarctic sea ice and underestimated westerly wind changes. Nat. Commun., 7, 10409, doi:10.1038/ncomms10409.

Rampal, P., J. Weiss, C. Dubois, and J.-M. Campin, 2011: IPCC climate models do not capture Arctic sea ice drift acceleration: Consequences in terms of projected sea ice thinning and decline. J. Geophys. Res., 116, C00D07, doi:10.1029/ 2011JC007110.

Richardson, M., K. Cowtan, E. Hawkins, and M. B. Stolpe, 2016: Reconciled climate response estimates from climate models and the energy budget of Earth. Nat. Climate Change, 6, 931935, doi:10.1038/nclimate3066.
Rosenblum, E., and I. Eisenman, 2016: Faster Arctic sea ice retreat in CMIP5 than in CMIP3 due to volcanoes. J. Climate, 29, 9179-9188, doi:10.1175/JCLI-D-16-0391.1.

Santer, B. D., and Coauthors, 2008: Consistency of modelled and observed temperature trends in the tropical troposphere. Int. J. Climatol., 28, 1703-1722, doi:10.1002/joc.1756.

Screen, J. A., and J. A. Francis, 2016: Contribution of sea-ice loss to Arctic amplification is regulated by Pacific Ocean decadal variability. Nat. Climate Change, 6, 856-860, doi:10.1038/ nclimate3011.

_ , I. Simmonds, and K. Keay, 2011: Dramatic interannual changes of perennial Arctic sea ice linked to abnormal summer storm activity. J. Geophys. Res., 116, D15105, doi:10.1029/ 2011JD015847.

Stroeve, J., and D. Notz, 2015: Insights on past and future sea-ice evolution from combining observations and models. Global Planet. Change, 135, 119-132, doi:10.1016/j.gloplacha.2015.10.011.

$\longrightarrow$, and - 2016: Corrigendum to insights on past and future sea-ice evolution from combining observations and models [Glob. Planet. Change (2015) 119-132]. Global Planet. Change, 144, 270, doi:10.1016/j.gloplacha.2016.07.003.

—, M. M. Holland, W. Meier, T. Scambos, and M. Serreze, 2007: Arctic sea ice decline: Faster than forecast. Geophys. Res. Lett., 34, L09501, doi:10.1029/2007GL029703.

_ , V. Kattsov, A. Barrett, M. Serreze, T. Pavlova, M. Holland, and W. N. Meier, 2012: Trends in Arctic sea ice extent from CMIP5, CMIP3, and observations. Geophys. Res. Lett., 39, L16502, doi:10.1029/2012GL052676.

Swart, N. C., and J. C. Fyfe, 2013: The influence of recent Antarctic ice sheet retreat on simulated sea ice area trends. Geophys. Res. Lett., 40, 4328-4332, doi:10.1002/grl.50820.

,-- E. Eawkins, J. E. Kay, and A. Jahn, 2015: Influence of internal variability on Arctic sea-ice trends. Nat. Climate Change, 5, 86-89, doi:10.1038/nclimate2483.

Taylor, K. E., R. J. Stouffer, and G. A. Meehl, 2012: An overview of CMIP5 and the experiment design. Bull. Amer. Meteor. Soc., 93, 485-498, doi:10.1175/BAMS-D-11-00094.1.

Turner, J., T. J. Bracegirdle, T. Phillips, G. J. Marshall, and J. S. Hosking, 2013: An initial assessment of Antarctic sea ice extent in the CMIP5 models. J. Climate, 26, 1473-1484, doi:10.1175/ JCLI-D-12-00068.1.

—, J. S. Hosking, T. J. Bracegirdle, G. J. Marshall, and T. Phillips, 2015: Recent changes in Antarctic sea ice. Philos. Trans. Roy. Soc., 373A, 20140163, doi:10.1098/rsta.2014.0163.

Uotila, P., P. R. Holland, T. Vihma, S. J. Marsland, and N. Kimura, 2014: Is realistic Antarctic sea-ice extent in climate models the result of excessive ice drift? Ocean Modell., 79, 33-42, doi:10.1016/j.ocemod.2014.04.004.

Winton, M., 2011: Do climate models underestimate the sensitivity of Northern Hemisphere sea ice cover? J. Climate, 24, 39243934, doi:10.1175/2011JCLI4146.1.

Xie, S.-P., Y. Kosaka, and Y. M. Okumura, 2016: Distinct energy budgets for anthropogenic and natural changes during global warming hiatus. Nat. Geosci., 9, 29-33, doi:10.1038/ngeo2581.

Zunz, V., H. Goosse, and F. Massonnet, 2013: How does internal variability influence the ability of CMIP5 models to reproduce the recent trend in Southern Ocean sea ice extent? Cryosphere, 7, 451-468, doi:10.5194/tc-7-451-2013. 